\title{
Proposed Simplified Procedure for Safety Factor Scaling for Various Life Cycles
}

\author{
Eliezer Shamir \\ Shamir Posner Brown Construction Engineering LTD., Bnei Brak 51201, Israel
}

\begin{abstract}
It is common to assume that structures are designed in view of 50 year life cycle as per Euro-Code 2 and other codes. In special cases, structures are designed in view of longer life cycle, such as bridges, important infrastructure facilities, important religious structures or in case of extended returning period of seismic event or floods. Beside issues of durability and maintenance aspects, this involves also the need to cover the probability of exceeding characteristic design live loads during the extended period, while keeping the same levels of the accepted risk that were assumed by the various codes, as good enough for the standard 50 year life cycle. Bearing in mind that design procedures, formulations, materials characteristic strengths and partial safety factors are used for these structures as per the existing codes, scaling of partial safety factors, or alternatively an additional "compensating" factor is required. A simplified approach and procedure to arrive at a reasonable calibration of the code safety factors based on 50 years to compensate for an extended life cycle, based upon structural reliability considerations, is proposed.
\end{abstract}

Key words: Life cycle, code calibration, scaling, safety factors, target reliability factor.

\section{Introduction}

The basic principle underlining the theory of structural safety is that, at any time along the structure's life cycle, a sufficient level of safety is provided. This has to do with preventing local or wide-spread collapse and avoiding deformations large enough to risk the structural stability, aiming to reduce the possibility of casualties.

The vehicle which enables to fulfil safety requirements is limiting strains of concrete and steel, as well as movements of the structure's components, to such values that will ensure the above mentioned. Control of these parameters is achieved by following design guidance and the computational procedures indicated by the codes.

Israeli Standard 466 (buildings), following the EC-2 in format of LRFD (load resistance factored design), is using a partial safety factors system from which safety of each structural component is derived.

Corresponding author: Eliezer Shamir, structural engineer, research fields: bridges and structural design. E-mail: shamir@spb.co.il.
The complete safety of the structure is guaranteed if, in addition, any possibility of progressive collapse is prevented and the stability of the structure as a whole is assured.

A certain level of safety (and not necessarily sufficient) will be achieved if, at any point along the life cycle of the structure and in any section, the following exists:

$$
\frac{R_{u}}{L}=K>1
$$

where,

$R_{u}$ represents for ultimate capacity-resistance in its most general meaning;

$L$ represents for load effect-in its most general meaning;

$K$ represents for safety level.

The idea of "load" relates to the influence of all the loads and movements acting on the structure and it manifests in axial and shear forces, bending moments and twisting moments, and also in corresponding deformations and strains. The larger the ratio $K$ is, the bigger the safety level is.

Eq. (1) is of a probabilistic nature rather than a 
deterministic one, so it must be calibrated to accommodate for life cycles different from the standard 50 years, using probabilistic approach and techniques.

\section{Statistical Dependency of Structural Capacity (Resistance) and Actions}

Formulating the idea of safety using Eq. (1) is in the most general sense. And yet, its mathematical meaning is not simple at all, since the parameters $R_{u}$ and $L$ do not represent deterministic values and both are in fact familiar to us on statistical basis only.

The main factors influencing the true load effect $L$ are:

(1) magnitude of the loads acting during life-cycle of the structure (or magnitude of relative deformations between its components) and the probability of those magnitudes to occur;

(2) expected loading cases in the structure (forecast load combinations and the probability of their occurrence);

(3) types of loads likely to occur (concentrated, distributed, varying and dynamic) and the probability of their occurrence;

(4) geometry of the structure to include its rigidities and the distribution of masses throughout its components;

(5) interaction of structure and ground;

(6) to what extent, the structure analysed model represents the structure's behavior in reality;

(7) life cycle of the structure.

The first five factors are stochastic variables and their probability is expressed well enough by the normal probability density curve (although some of the loads are better represented by the Gumbel curve). The sixth factor is statistical yet biased, as it changes deliberately, always towards improving, following development of technology and research. Therefore, its probability density will be better expressed by a log-normal probability density curve. The structure's life cycle can be a pre-determined specific value.
The main factors influencing the ultimate capacity (resistance) are mainly:

(1) the structure's geometry and the geometry of the sections;

(2) methods, procedures staging and accuracy of execution;

(3) the actual rigidities of the various components and foundation;

(4) dispersal of the real strength properties throughout the structural components;

(5) the level of homogeneity of the structural components;

(6) the real strength of materials as tested in laboratory;

(7) to what extent and reliability, lab-testing of materials, representing their real behavior in situ, especially regarding the geometric difference between the structure and the sample, and due to dispersal of the material in the structure;

(8) to what extent and reliability, algorithms used for strength calculation representing behavior of the structure in reality.

The first five factors are stochastic variables and their probability density is expressed well enough by the normal curve. The other variables are better represented by a log-normal curve, as they are biased.

From the above mentioned we gather, probability-wise, the behavior of the load effect is represented well enough by a normal (symmetric) curve and that the probable behavior of the capacity is well represented by a log-normal (a-symmetric) curve. But for simplicity, it is accepted by the codes as "good enough" to assume for capacity also a normal distribution.

Since the values of $R_{u}$ and $L$ are probabilistic functions, it is clear that Eq. (1) is showing a probabilistic behavior.

\section{The Safety Function and the Safety Level}

A safety function $G$ may be written in a few ways: 


$$
\begin{gathered}
G=\left[\frac{R_{u}}{L}\right]>1 \\
\text { or } G=\left[R_{u}>L\right] \\
\text { or } G=\left[R_{u}-L\right]>0
\end{gathered}
$$

Due to the probabilistic nature of the load and capacity functions and in order to obtain a defined level of safety stored in a calculated section, it is easier to express the safety function $G$ as a subtractive function as Eq. (2) above.

A conceptual graphic description of the safety function $G$ may be found for example in fib Model Code [1], or in IABSE (International Association of Bridge and Structures Engineering) Introduction to safety and reliability of structures [2].

The issue of probabilistic approach to structural safety was thoroughly investigated by many researches and summarized (temporarily) in JCSS (Joint Committee on Structural Safety) Draft Model Code [3]. It was found that the practical, simple and good enough way to express the reliability level will be by the $\beta$ factor, developed while assuming a normal probability distribution behaviour for both resistance and load effects.

In order to reach a common ground to this discussion, the main and basic issues related to the probabilistic approach to safety are summarized hereafter.

For a normal probability distribution of both resistance and load effect, the reliability is expressed by:

$$
\beta=\frac{R_{m}-L_{m}}{\left(\sigma_{R}^{2}+\sigma_{L}^{2}\right)^{\frac{1}{2}}}
$$

where,

$\beta$ represents for reliability factor;

$L_{m}$ and $R_{m}$ represent for the average values of capacity (resistance) and load;

$\sigma_{L}$ and $\sigma_{R}$ are the standard deviations of capacity and load.

Solutions for situations where one function is of normal nature while the other is of log-normal nature, or where both are of log-normal nature, may be found for example in Ref. [4].

As it is easily shown in the documents mentioned above, the cumulative probability is the value of $\Phi$ for $(-\beta)$, that is:

$$
P_{f}=\Phi(-\beta)
$$

as shown in Fig. 1.

From Eq. (3), we obtain that the required average resistance is:

$$
R_{m}=L_{m}+\beta \cdot\left(\sigma_{R}^{2}+\sigma_{L}^{2}\right)^{1 / 2}
$$

The second term of Eq. (5) can be easily identified as the strength component which provides us the required safety, assuming we know the value of $\beta$, and if we know the standard deviations of the capacity and load.

To get an idea of the level of overall safety, it is well common to define $\alpha_{L}$ and $\alpha_{R}$ as follows:

$$
\alpha_{R} \geq \frac{\sigma_{R}}{\left(\sigma_{R}^{2}+\sigma_{L}^{2}\right)^{1 / 2}}, \alpha_{L} \geq \frac{\sigma_{L}}{\left(\sigma_{R}^{2}+\sigma_{L}^{2}\right)^{1 / 2}}
$$

Therefore, these expressions show the "weight" of the standard deviation of the capacity and load in relation to the standard deviation of the safety function.

Using very simple algebraic manipulations while C.O.V. (coefficient of variations) are defined as:

$$
V_{R}=C . O \cdot V_{\cdot(R)}=\frac{\sigma_{R}}{R_{m}}, V_{L}=C . O \cdot V_{\cdot(L)}=\frac{\sigma_{L}}{L_{m}}
$$

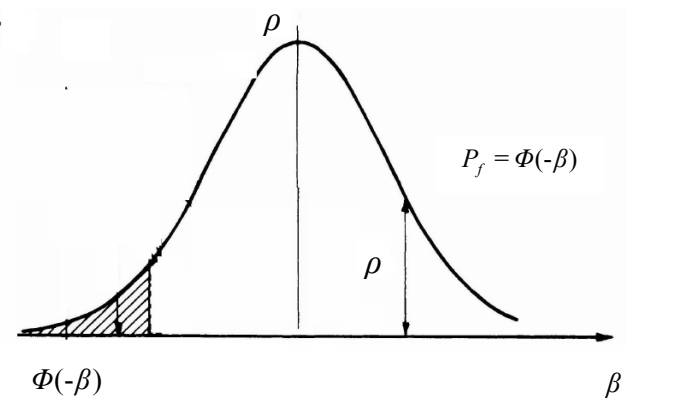

Fig. 1 The probability density function of the safety function. 
We obtain:

$$
R_{m}-L_{m}=\beta \cdot\left(\alpha_{R} \cdot \sigma_{R}+\alpha_{L} \cdot \sigma_{L}\right)
$$

And the overall safety coefficient becomes:

$$
K=\frac{R_{m}}{L_{m}}=\frac{1+\alpha_{L} \cdot \beta \cdot V_{L}}{1-\alpha_{R} \cdot \beta \cdot V_{R}}
$$

The safety coefficient according to Eq. (6) is an overall safety factor, used for design in the L.F.D (load factor design).

For the LRFD (load resistance factored design) approach, a little more sophistication is required. Characteristic values, $L_{C}$ and $R_{C}$ for design are defined. These relate to the true average values of strength of materials and load effects in each of the possible actions (bending, axial load, shear and torsion), and for various construction types, while keeping a certain small safe margin distance from them.

Since it is assumed for sake of simplicity that both capacity and load behave as normal probability functions, we can use the theory of probability to determine the limit state values to which we should refer as follows:

$$
\begin{aligned}
& R_{c}=R_{m}-n_{R} \cdot \sigma_{R} \\
& L_{c}=L_{m}+n_{L} \cdot \sigma_{L}
\end{aligned}
$$

where, $n_{L}$ and $n_{R}$ represent for the number of standard deviations to keep the limit state value remote from the average value.

It is quite easy to show, using simple algebraic manipulations, that, at the point of design where the probability of failure is maximal, design values for capacity and load determined by theory of safety must exist, according to Eq. (5), as follows:

The maximal load possible:

$$
L_{d}=L_{m}+\alpha_{L} \cdot \beta \cdot \sigma_{L}
$$

The maximal capacity possible:

$$
R_{d}=\mathrm{R}_{\mathrm{m}}-\alpha_{R} \cdot \beta \cdot \sigma_{R}
$$

and to maintain safety:

$$
R_{d}>L_{d}
$$

And we should define the partial safety factors by:

$$
\begin{gathered}
L_{d}=L_{c} \cdot \gamma_{L} \\
R_{d}=\frac{R_{c}}{\gamma_{R}}
\end{gathered}
$$

We obtain the partial safety coefficients as follows:

$$
\begin{gathered}
\gamma_{L}=\frac{\alpha_{L} \cdot \beta \cdot V_{L}+1}{n_{L} \cdot V_{L}+1} \\
\gamma_{R}=\frac{1-n_{R} \cdot V_{R}}{1-\alpha_{R} \cdot \beta \cdot V_{R}}
\end{gathered}
$$

Parameters are detailed as in Eqs. (6), (7) and (8).

The coefficient $\gamma_{R}$ is calculated for each and every type of capacity (bending, shear, torsion and axial force) and for various components (beam, column, etc.).

Thus, it is clearly demonstrated that partial safety factors are not an absolute deterministic value, but rather of a probabilistic nature.

In the literature dealing with the theory of safety, we find accepted and proposed representative values for standard deviations and for bias factors, and coefficients of variation (C.O.V.), which are typical for various structure components and various loads. Many researches and investigations were (and still are) conducted, aiming to define those parameters, among which we shall point out as examples the JCSS proposal for structural reliability codes for probabilistic design [3], or the works of Nowak and Szerszen [5] or Frangopol [4].

An example for such data is shown in Table 1 (after A.S. Nowak).

The overall expression of the component (or structure) capacity will, in fact, take the form:

$$
R=R_{n} \cdot M \cdot F \cdot P
$$

where,

$R_{n}$ represents for calculated algorithm of minimal capacity for component or structure;

$M$ stands for expresses influence of materials;

$F$ stands for expresses influence of execution;

$P$ is expresses professional influences (design).

When dealing with structural capacity, the $R_{n}$ (resistance) factor includes the function of structural strength, which takes into account non-linear behavior, including the sequential formation of plastic hinging, co-existing and performing, all together, until a mechanism is reached. 
Table 1 Probabilistic sample data for structural components, after A.S. Nowak and Szerszen [5].

\begin{tabular}{lll}
\hline Component and load & $\gamma_{R}$ & C.O.V. \\
\hline Pre-stressed concrete beam, cast in situ (bending) & 1.114 & 0.119 \\
Pre-stressed concrete beam, cast in situ (shear) & 1.159 & 0.120 \\
Pre-stressed beam, industrial product (bending) & 1.084 & 0.081 \\
Pre-stressed beam, industrial product (shear) & 1.130 & 0.105 \\
Solid plate, cast in situ (bending) & 1.052 & 0.169 \\
Pre-stressed solid plate, industrial product & 1.053 & 0.070 \\
Pre-stressed solid plate, cast in situ & 0.960 & 0.146 \\
Columns with hoops, cast in situ & 1.107 & 0.136 \\
Columns with spiral, cast in situ & 1.163 & 0.124 \\
\hline
\end{tabular}

Table 2 Probabilistic data for loads, after Frangopol [4].

\begin{tabular}{|c|c|c|c|c|}
\hline & \multicolumn{2}{|c|}{ Maximal loads } & \multicolumn{2}{|c|}{ Maximal loads for 50 years period } \\
\hline & Bias factor $\lambda_{L}$ & C.O.V. & Bias factor $\lambda_{L}$ & C.O.V. \\
\hline Self weight (cast in situ) & 1.05 & 0.1 & 1.05 & 0.1 \\
\hline Self weight (factory cast) & 1.03 & 0.08 & 1.03 & 0.18 \\
\hline Useful load & 0.24 & 0.65 & 1.0 & 0.18 \\
\hline Wind & & 0 & 0.78 & 0.37 \\
\hline Earthquake & 0 & 0 & 0.66 & 0.56 \\
\hline
\end{tabular}

In the mentioned literature above, we can find C.O.V. values and standard deviations for each parameter separately, but for this discussion, we use the values in Table 1 which express the combined C.O.V. of the capacity and include all those influences all together at sufficient and "good enough" approximation.

Table 2 contains some accepted representation magnitudes of bias factors and the variation factors of the loads based on the mentioned sources above. Naturally, useful loads may be attributed to a wider range of values according to the load type and its characteristics. The values in the table are just an example.

The bias factor is defined in probability theory as the ratio between the average value and the nominal (characteristic) value represented by this average:

$$
\text { Bias }=\lambda_{X}=\frac{X_{m}}{X_{n}}
$$

Values for $\alpha_{L}$ and $\alpha_{R}$ are obtained by a process of experiment and tuning of existing standards using the experiments results. The accepted values found in international various standards vary from 0.6 to 0.8 . Since the condition $\sigma_{R}^{2}+\sigma_{L}^{2} \geq 1$ is the minimal required condition, it is customary, according to JCSS [3], and in order to be on the safe side and to simplify calculations, to use the following values:

$$
\alpha_{L}=0.7, \alpha_{R}=0.8
$$

\section{Target Reliability Factor $\beta$ as Related to Structural Life Cycle (Proposed Simplified Approach)}

The values of the reliability factor $\beta$ are derived from the accepted levels of risk. This issue is treated extensively in various sources, mainly in the JCSS proposal for probabilistic standard [3], also in the fib Model Code [1], and the preface to the EC-2 standard and also by Nowak [5], Schneider [2] and others. The recommended values of $\beta$ for establishing safety factors are defined as "reliability target values".

Target values are at times chosen either according to importance level of the structure or how it is populated, and/or the consequences of its failure. Sometimes, it is chosen according to the type of failure and its circumstances (level of ductility, designed robustness, alternative static systems, elastic behavior, etc.), and sometimes as a function combining all of the above. Target values are given 
for the agreed value of tolerable annual human lethal probability due to failure.

The variability range of target values according to annual failure probability is usually in the range of $\beta=$ 3.0-6.0, when the lowest value is for structures whose higher probability of failure is accepted and agreed upon. The lower the value of $\beta$, the higher the probability of failure. Further detailing of accepted target values of $\beta$, the ultimate and service limit states, may be found in the sources above.

According to Eq. (4), the annual probabilities of failure for the above range are approximately:

$$
P_{f}=1.3 \times 10^{-3} \text { to } 1.3 \times 10^{-7}
$$

In order to tune the $\beta$ factor, it was decided and recommended by fib that, as a rule, the usual case will be a design in view of a 50-year life cycle and this will be used to establish target values of failure probability. In case of special structures, it is customary to consider a longer life cycle. For instance, the Israeli Standard for bridges considers 120 years, following the British Standard BS-5400. The AASHTO (American Association of State Highway and Transportation Officials) Standard for bridges considers a life cycle of 75 years, while the EN 1991-2 considers 100 years.

Knowing an agreed accepted annual probability of failure, it is now easy to estimate the probability corresponding to different life cycle according to Eq. (19), but dealing with low probabilities as in this case, it is possible to calculate the probability of failure in 50 years at a sufficient approximation as 50 times the annual probability (at a deviation of less than 1\%), that is:

$$
P_{f T=50}=50 \times P_{f T=1}
$$

According to Eqs. (4) and (16), the magnitudes of $\beta$ for a 50 years life cycle will usually be in the range of $\beta=1.5-4.5$ approx

\section{Partial Safety Factors in Standardization}

According to Eqs. (12) and (13), the numeric value of the partial safety factor is a function of the probabilistic parameters mentioned in Section 3 and it has a probabilistic value in itself.

In order to be able to use partial safety factors in a practical manner, these cannot be left to be determined individually by each and every designer and for each and every situation and structural scheme. It is necessary to establish and determine a mandatory framework of specific partial safety factors that will eventually yield the target safety level.

In order to create a uniform format and a uniform acceptable safety level, standardization dictates minimal values for these factors. The values dictated by the standard are absolute values, not probabilistic, established by Eqs. (12) and (13) while the values set in them are typical of each of the probabilistic parameters. To do so, standardization assumes several basic assumptions which manifest in these values.

These basic assumptions and their influence on standard deviations and coefficient of variation are not in this article's scope. It is noted, however, that these assumptions address each of the factors influencing capacity and load mentioned above, and also the mechanism of action and behavior of components' sections in a state of real ultimate failure, as in a state of design ultimate limit state. It also relates to the parameters $\alpha_{L}, \alpha_{R}$ and $\beta$, as specified in Section 3.

\section{Calibration of Partial Safety Factors That Exceed Basic Assumptions}

Giving absolute deterministic values to standard safety factors, as per Section 5, turns the formulation of the safety issue from a "probabilistic case" into a "private determinated case", in which certain basic assumptions hold, resulting in absolute values for the factors in Eqs. (12) and (13).

Therefore, the partial safety factors in the codes will hold as long as the basic assumptions are fulfilled

But as we know, these assumptions do not always fully take place. In such cases, we need to introduce a correction in order to compensate for the deviations from those basic assumptions. Such factors exist in 
codes and are called "behavior factors" or "calibration factors" or such. Typical cases where such factors are used are: punching shear calculation, cases of potential brittle failure or possibility for progressive failure, certain cases of successive failure path (unlike parallel failure path). All the cases mentioned above influence basic assumptions made in regards to standard deviations and C.O.V.

A less common case for altering basic assumptions is the change in structure's life cycle to be considered in safety calculations. Here, we deal with the basic assumptions that were made in regard to the agreed (and tolerated-acceptable) probability of failure, meaning, in fact, the target value of reliability factor $\beta$. Such cases occur when dealing with structures of utmost importance, or highly valuable structures, or in case of outstanding consequences in case of failure, and/or a demand for exceptionally long existence of a structure.

Regarding the statement above for approximation as in Eq. (16), we should want the same failure probability $P_{f T=50}$ (established by fib recommendations for a 50 years life cycle) to be kept also for the longer life cycle $T$, then the annual failure probability that must exist in such a case approximately:

$$
P{ }_{f T=1}=\frac{P_{f T=50}}{T}
$$

Since the partial safety factors that exist in codes are established according to failure probability in a 50 years life cycle and we are going to use the same codes to design the extended life cycle constructions (using the same set of partial safety factors), the probability of failure for a 50 years period should be determined by the annual probability of failure $P_{f T=1}^{*}$ which we should consider due to the longer period. This will enable to determine the needed change in the reliability factor $\beta$.

This is of course a smaller value compared to the recommended value by fib for the standard 50 year structure.
Thus, the resulting amended probability of failure for a 50 years period which is approximately:

$$
P_{f T=50}^{*}=P_{f T=1}^{*} \times 50=\Phi\left(-\beta_{50}^{*}\right)
$$

According to the probability obtained in Eq. (18), we can establish the amended target value of $\beta_{50}^{*}$, complying with this probability, to be used in calibrating the partial safety factor for load and materials and in establishing the needed correction factor. From hereon, we can calculate construction according to the code strength values and partial safety factors, all of them changed by the correction factor established according to the amended target value of $\beta_{50}^{*}$.

\section{Updating Partial Safety Factors for Seismic Design of Bridges}

To exemplify the influence of the simplified approach above, as well as of the change of structure life cycle, on the partial safety factors, an analysis of the seismic bridge design will be demonstrated.

Bridges are designed in Israel for a probability of failure in view of a 120 years life cycle following the British Standard BS-5400. And yet, the construction is designed in compliance with Israeli Standard IS-466, the latter being calibrated to a 50 years life cycle in view of the $\beta$ target reliability as per EC- 2 and fib Model Code.

IS-466 determines the partial safety factor for seismic loads in structures to be $\gamma_{L}=1.0$ and the strength is calculated using the design strengths stated in IS-466. In the correction addendum No.1 for IS-1227 for Bridge Loads, it is stated that the partial safety factor for seismic loads will be $\gamma_{L}=1.0$ for bridges as well.

Since bridges are designed in compliance with IS-466 design values and factors - this contradiction clearly calls for calibration. IS-1227 must ensure that the level of safety required for buildings, for a 50 years life cycle, is kept as a minimum for bridges as well, but for a 120 years period. Thus, there is a clear deviation from the code basic assumptions, and the 
partial safety factors must be recalibrated.

For this purpose, we re-establish the probabilistic parameters in Eqs. (12) and (13) for partial safety factors.

\subsection{Seismic Load}

The standard seismic load is derived from an earthquake having $10 \%$ probability being exceeded in 50 years, meaning a fractile of $10 \%$. For simplicity, the parameter $n_{L}$ in Eq. (8) is calculated assuming a normal destribution function, in which: $\Phi\left(-n_{R}\right)=0.1$, thus: $n_{L}=1.282$.

For seismic load, we assume, according to Table 2:

$$
\begin{gathered}
V_{L}=C . O . V_{L}=0.56 \\
\sigma_{L}=V_{L} \cdot L_{m}-0.56 L_{m} \\
\lambda_{L}=(\text { Bias })=0.66
\end{gathered}
$$

There are now two possible approaches to be considered in order to determine the seismic load for 120 years period.

\subsubsection{Approach 1}

The seismic load will be determined, here as well, for an earthquake that there is a probability of $10 \%$ being exceeded in 50 years time, regardless of the structure life cycle structure.

\subsubsection{Approach 2}

The seismic load will be determined for an earthquake that there is a probability of $10 \%$ being exceeded during the structure's life cycle which is now taken as 120 years.

The load should be determined according to Section 7.1.1 - it means an annual probability of about 0.002 for such an event during the 50 years of the structure life cycle. In this case, the seismic load remains unchanged and, as per Eq. (19), we obtain that the probability for such a load during a 120 year period is about $22 \%$.

If the load should be determined according to possibility according to Section 7.1.2, we obtain the load for an earthquake for which there is a probability of $10 \%$ being exceeded in a 120 years. Its returning period:

$$
\begin{gathered}
T=\frac{1}{1-(1-p)^{1 / t}}= \\
\frac{1}{1-(1-0.1)^{1 / 120}}=1,140 \mathrm{y}
\end{gathered}
$$

The change in the load magnitude, as a result, should be obtained from the National Seismologic Institution according to the characteristics of earthquakes in Israel.

But one may use as a reasonable approximation, following EC-8, a calibration factor to the code design load derived from 475 year return period, as per:

$$
N=\left(\frac{T}{475}\right)^{0.35 \div 0.45}
$$

$N$ is ranging between 1.35 to 1.48 (approx.) and an average $N=1.4$ will be a reasonable assumption.

\subsection{Safety Function}

According to Eq. (3) instead of relating to different safety factors for different loads, we will assume that we deal with an average capacity. After Frangopol recommendation (1999), since the seismic capacity of bridges, is usually governed by column capacity for combined axial load and bending, we should use average values from Table 1 to represent the capacity:

$$
\begin{aligned}
& V_{R}=\text { C. } O . V_{R}=0.135 \\
& \lambda_{R}=(\text { Bias })=1.12
\end{aligned}
$$

And thus: $\sigma_{R}=R_{m} \cdot V_{R}=0.135 R_{m}$.

We select values for earthquake from Table 2 and given the $\lambda$ factors above corresponding with capacity and load, we approximately obtain:

$$
\beta=\frac{1.1 R_{u}-0.6 L}{\left(0.02 R_{u}{ }^{2}+0.1 L^{2}\right)^{1 / 2}}
$$

Compliance with this condition greatly represents, in fact, the safety level that currently exist in the Israeli Code Design.

\subsection{Establishing a Calibrated Target Value for $\beta_{50}^{*}$}

IS-466 calculates $L_{d}$ using a safety factor of $\gamma_{L}=1.0$. According to Eq. (12), also using $\alpha_{L}=0.7$ as recommended, partial safety factor for loads becomes: 


$$
1.0=\frac{0.7 \beta \cdot 0.56+1}{1.282 \cdot 0.56+1}
$$

resulting in $\beta=1.83$ (taking into account a 50 years life cycle).

As per Eq. (4), the corresponding $\Phi\left(-n_{R}\right)$ of failure for this $\beta$ is:

$$
\Phi(-1.83)=P_{f T=50}=0.0336
$$

Since IS-466 was written assuming a 50 years safety life cycle, the annual failure probability is about:

$$
P_{f T=1.0}=67.2 \times 10^{-5}
$$

Yet, in order to sustain the same safety level for a 120 years period, according to Eq. (17), we should maintain an annual probability of failure of:

$$
P_{f T=1.0}^{*}=28 \times 10^{-5}
$$

Since construction is designed according to IS-466, in which all safety factors are for 50 years period, we obtain an equivalent failure probability for 50 years of:

$$
P_{f T=50}^{*}=28 \times 10^{-5} \times 50=0.014
$$

which means according to Eq. (4):

$$
\Phi\left(-\beta_{50}^{*}\right)=0.014
$$

And the corresponding equivalent reliability factor becomes:

$$
\beta_{50}^{*}=2.195
$$

\subsection{Calculation of Correction Factors for Loads and Capacity}

Introducing the $\beta_{50}^{*}$ above in Eq. (12), we get the partial safety factor for load:

$$
\gamma_{L}^{*}=\frac{0.7 \times 2.195 \times 0.56+1}{1.282 \times 0.56+1}=1.083
$$

A similar procedure is made now for the material partial safety factor.

When dealing with strength of materials, it is customary to use a probability of $5 \%$ for deviations (fractile $=5 \%$ ), and assuming a behavior of normal probability distributions, this case yields $n_{R}=1.645$. According to Table 1, we may select a representing C. $O . V_{R}=0.135$ and $\lambda_{R}=0.8$ as recommended.

When set in Eq. (13), this yields:

$$
\gamma_{R}^{*}=\frac{1-1.645 \times 0.135}{1-0.8 \times 2.195 \times 0.135}=1.018
$$

If we do not wish to alter the material safety factor, while we keep calculating the section strength using the same design stresses of IS-466, we must increase the safety factor for seismic loads through multiplying by a calibration factor of:

$$
\gamma_{T=120}^{*}=\gamma_{L}^{*} \cdot \gamma_{R}^{*}=1.083 \times 1.018=1.10
$$

\section{Final Results and Discussion}

If we were to determine the seismic load according to the consideration in the aforementioned possibility in Section 7.1.2 while using IS-466 and IS-1227 to calculate seismic load, we should multiply this design load by a partial safety factor which expresses both the increase of the characteristic magnitude of the load as per $N$ from Eq. (20), and the increase of the partial safety factor, derived from altering the life cycle. That is:

$$
\gamma_{L}^{*}=\gamma_{T=120}^{*} \cdot N \cdot \gamma_{L \text { code }}=1.10 \times 1.41 \times 1.0=1.55
$$

(instead of $\gamma_{L}=1.0$ as stated in IS-1227).

According to Eq. (20), multiplying the standard load by a calibration factor of 1.55 yields a load identical to that of an earthquake having a return period of 1,400 years compared to the standard 475 years event.

This procedure is recommended when highly important bridges are of concern, or is case where very high consequences of failure are involved.

However, as it is customary to determine the seismic load by the consideration in the aforementioned possibility in Section 7.1.1, meaning according to $10 \%$ probability of exceeding in a period of 50 years (regardless of the structure life cycle), and the load is calculated according to IS-1227, we must as a minimum, multiply that standard load by a load calibration factor for:

$$
\gamma_{L}^{*}=\gamma_{T=120}^{*} N \cdot \gamma_{L \text { code }}=1.10 \times 1.0 \times 1.0=1.10
$$

According to Eq. (20), multiplying the standard load by a calibration factor of 1.10 yields a load identical to that of an earthquake having a return 
period of 603 years compared to the standard 475 years event.

\section{Conclusions}

A simplified approach and procedure are proposed to enable an easy calibration of partial safety factors due to change in life cycle of structure from the standard 50 year life cycle usually assumed by design codes for regular structures.

In the case that the structure is designed to a specially dedicated standard, safety factors and design loads are already pre-defined for use as indicated by that specific standard and no further calibration are required. But in the case that no specific dedicated standard is available, and still due to its importance or cost or complexity, etc., the structure is to be designed in view of an extended life cycle (longer than the "usual" 50 years considered for regular structures), this simplified procedure provides an easy way to calibrate partial safety factors, which, combined with calibration of design loads of a stochastic nature, will enable a proper and safe design while still applying the "usual" standard, aiming at 50 years life cycle.

This is the case of the seismic design of bridges in Israel, shown as an example above.

\section{References}

[1] Model Code 2010 (Final draft), Fib, Switzerland, 2011.

[2] J. Schneider, Introduction to Safety and Reliability of Structures, IABSE (International Association for Bridge and Structural Engineering), Zurich, 1997.

[3] Probabilistic Model Code (12th draft), Joint Committee on Structural Safety, 2000.

[4] D.M. Frangopol (Ed.), Bridge Safety and Reliability, TG300.B755-1999, Contributions by: F. Mosey, M. Ghosn, A.S. Nowak, D.M. Frangopol, G. Hearn, Structural Engineering Institute of the American Society of Civil Engineers, Virginia, 1999.

[5] A.S. Nowak, M.M. Szerszen, Calibration of design code for buildings (ACI 318), parts 1\&2, ACI (American Concrete Institute) Structural Journal 100 (3) (2003) 377-382. 